\title{
Effect of Variable Cyclic Prefix Length on OFDM System Performance over Different Wireless Channel Models
}

\author{
Amar A Al-jzari ${ }^{1}$, Ivica Kostanic ${ }^{1}$, Khalid Hassan Mohamed Mabrok ${ }^{2}$ \\ ${ }^{1}$ Department of Electrical and Computer Engineering, Florida Institute of Technology, USA \\ ${ }^{2}$ Department of Electronic Engineering, University of Garden City, Khartoum, Sudan
}

Copyright (C) 2015 Horizon Research Publishing All rights reserved.

\begin{abstract}
A Cyclic Prefix (CP) is a significant feature of an OFDM waveform. It is used to completely eliminate both Inter-Symbol Interference (ISI) and Inter-Carrier Interference (ICI) as long as the CP length is greater than the channel delay spread. By eliminating both ISI and ICI, the $\mathrm{CP}$ compensates for the effect of the multipath dispersion; but it consumes a considerable amount of the scarce spectrum and the power. Conventional OFDM uses a fixed and large CP length to overcome the ISI caused by channel delay spread under wireless mobile environment. This approach may degrade the overall spectral efficiency as well as consumes relatively more transmitter energy. Therefore, there is a need to adopt the $\mathrm{CP}$ length according to instantaneous channel parameters. In this paper, we presented a method for investigating the effect of varying the $\mathrm{CP}$ length on OFDM system performance over different multipath channel models. We estimated the variable CP length based on the RMS delay spread of the channels' power delay profile (PDP). According to this method, the estimated CP length optimizes the system capacity and improves the overall system performance. We showed that this approach could lead to a mathematical formula presenting the relationship between the $\mathrm{CP}$ length and the RMS delay spread of the channels.
\end{abstract}

Keywords Cyclic Prefix Length, OFDM System, Power Delay Profiles, RMS Delay Spread, System Capacity

\section{Introduction}

Orthogonal Frequency Division Multiplexing (OFDM) has become the most popular transmission technique for wireless communication over wide band channels that exhibit frequency selectivity. In broadband wireless communication, the delay spread caused by multipath fading channel will usually introduce ISI to the receiver. OFDM transmission scheme was devised to improve radio link performance degraded by the multipath fading. Due to its robustness against multipath fading the OFDM is becoming a modulation technique of choice for most commercial high-speed broadband wireless communication systems. The OFDM is a special form of multi-carrier modulation schemes that is capable of overcoming the frequency selectivity of the radio channels and providing the high data rates without ISI [1-5].

A circular extension known as the $\mathrm{CP}$ is generally inserted in OFDM transmission to eliminate both ISI and ICI resulting from the multipath nature of the physical channel. To achieve this goal, the $\mathrm{CP}$ length must be greater than the delay spread of the multipath channel [6]. However, the introduction of the $\mathrm{CP}$ reduces the bandwidth efficiency and decreases the data rate (system capacity) because it conveys no information. It also disperses the transmitter energy (the amount of consumed power depends on how large the $\mathrm{CP}$ length), where the signal-to-noise (SNR) lost due to the CP introduction indicates the loss of transmission energy. Because of the loss of SNR and the bandwidth efficiency, the CP length needs to be chosen optimally. Typically, it is chosen based on the multipath channel duration in a given operating environment $[7,8]$.

The CP is always appended to the head of the OFDM symbols. This result is in loss of spectral efficiency of the system. For example, in IEEE $802.11 \mathrm{a} / \mathrm{g}$ standard about $25 \%$ of the useful signal is utilized by the CP $[9,10]$. Therefore, it is essential to understand the $\mathrm{CP}$ adaptation scheme. Conventional OFDM system uses a large and fixed CP length, which is selected to be several times more than the RMS delay spread of the operating environment. This CP selection mandates devices which experience smaller RMS delay spread to use an excessively longer $\mathrm{CP}$. In this situation, the scarce network resource is misused and the limited battery power is wasted from the device perspective $[9,10]$. This fact motivates the researchers into ways for making the $\mathrm{CP}$ length shorter and even adaptive. This background encourages them to use an adaptive and variable $\mathrm{CP}$ length which can introduce a significant enhancement in spectral efficiency. In 2004, Zhang [11] proposed a method to adopt the CP length based on the variation of the channel delay spread. His method suggested selection of the CP length which is twice the channel RMS delay spread. The main drawback of this approach is that a fixed CP length, which is impractical especially for mobile applications. This 
fact is also motivated the use of variable CP length. In 2005, Chiwoo, Youngbin, Panyuh, Hyeonwoo and Jaeweon [12] proposed another method to adopt the CP length via adjusting the sampling rate. The drawback of such as solution is increased signaling overhead. In $[11,12]$ approach, the Power Delay Profile (PDP) of the channels is fitted into a Negative Exponentially Decaying Profile (NEDP). Based on the NEDP a mathematical formula is derived to estimate the variable CP length for the different channel models. The evaluation of the $\mathrm{CP}$ length requirements for different mobile environments is the problem addressed in this research. In this paper, we proposed an approach which can be utilized to enhance the method developed in $[11,12,15]$. We presented a method to estimate the variable CP length based on the RMS delay spread of the channels' PDP, where the channels' PDP are not fitted into NEDP but it is scaled by a weighting function to ensure the synchronicity assumption. Furthermore, an analytical method for evaluating Signal to Interference and Noise Ratio (SINR) in OFDM system is utilized in this paper to evaluate the optimum $\mathrm{CP}$ length over different channel model.

\section{System Model Description}

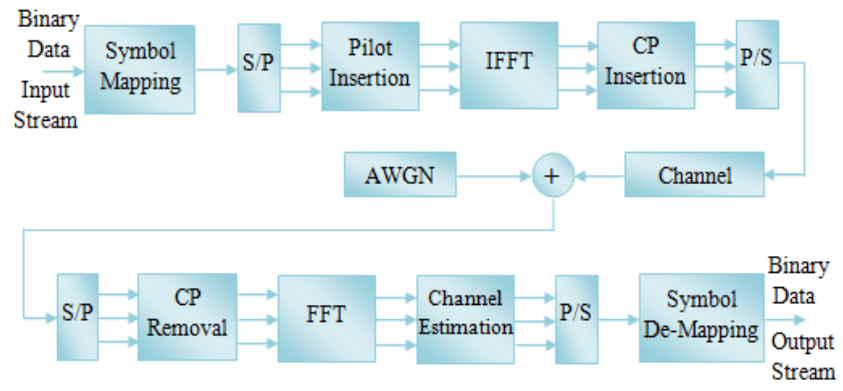

Figure 1. Block diagram of OFDM system

Fig. 1 shows a basic model of OFDM system. First the input binary data are generated and converted to the data symbols by using different modulation schemes such as BPSK, QPSK and QAM etc. Once the sequence of binary values is mapped to a data symbol form, the next step is to generate an OFDM waveform by converting the serial data symbol sequence into a number of shorter parallel symbol sequences. These modulated signals are processed by Inverse Fast Fourier Transform (IFFT) block to transfer the signal from the frequency domain to the time domain. Then, the CP is added to the signals. This CP extends the OFDM symbol by copying the last samples of the OFDM symbol and appending it in front of the transmitted OFDM symbol. The reason for that is to preserve the orthogonality between the sub-carriers, and to allow the receiver to integrate over an integer number of sinusoid cycles for each of the multipath when it performs OFDM demodulation with Fast Fourier Transform (FFT). After adding the CP, the signals are converted to serial form and transmitted through the channel [13]. At the receiver side, a reverse process is performed.
The serial data is received and converted to the parallel form. Then the CP is removed. After removal of CP, the FFT is performed to convert the signals to frequency domain. Then the signals are demodulated using different modulation schemes to get the original binary data [13].

Fig.2 shows the proposed OFDM system model. According to this model, at the transmitter end the signal is modulated and subsequently the CP controller attaches the CP length adaptively to mitigate both ISI and ICI introduced due to the multipath fading channel. At the receiver end, the $\mathrm{CP}$ controller detaches the CP length before demodulator in order to recover the transmitted signal.

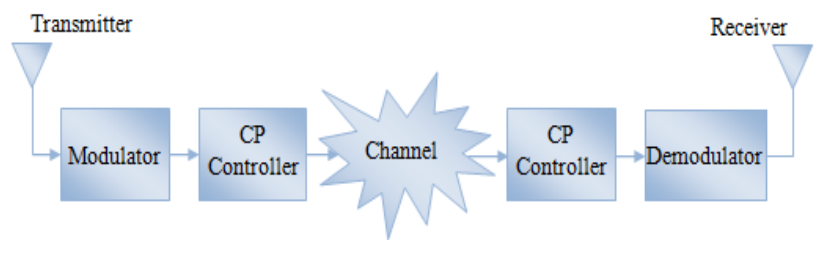

Figure 2. Block diagram of proposed OFDM system

\section{SINR Calculation Method}

In this section, we presented an analytical method for evaluating SINR in OFDM system as utilized in [14]. The SINR expression is used in our analysis as function of CP length, PDP, symbol length and thermal noise power. The useful and the interference signal powers are both obtained from the channels' PDP scaled by a weighting function called "bias function" given as:

$$
C(\tau)=\left\{\begin{array}{cc}
0, & \tau<0 \\
1, & 0 \leq \tau<T_{g} \\
0, & T_{O F D M} \leq \tau \\
\frac{T_{u}-\left(\tau-T_{g}\right)}{T_{u}}, & T_{g} \leq \tau<T_{g}
\end{array}\right.
$$

Where $T_{g}$ is the CP length, $T_{u}$ is the useful OFDM symbol length and $T_{O F D M}$ is the total OFDM symbol length which is a combining length of the CP and the useful OFDM symbol.

The channel PDP is assumed to have $P$ taps and complex gain with power $\left|\alpha_{i}\right|^{2}$ and $i^{\text {th }}$ tap at delay $\tau_{i}$. The channel is normalized such that:

$$
\sum_{i=1}^{p} a_{i}=1
$$

The SINR per subcarrier due to this static channel, with transmit signal power of $\sigma_{x}{ }^{2}$ per subcarrier and thermal noise of variance $\sigma_{n}{ }^{2}$ per subcarrier, can be written in terms of useful signal and interference signal power as:

$$
S I N R=\frac{p_{S}}{P_{i}+\sigma_{\omega}{ }^{2}}
$$

Where $P_{s}$ is the useful signal power, $P_{i}$ is the interference signal power and $\sigma_{\omega}{ }^{2}=\sigma_{n}{ }^{2} / \sigma_{x}{ }^{2}$ is the variance of the additive white noise .

The useful signal power is given by:

$$
P_{s}=\sum_{i=1}^{p} C(\tau)^{2}\left|a_{i}\right|^{2}
$$


The interference signals power is given by:

$$
P_{i}=\sum_{i=1}^{p}\left(1-C(\tau)^{2}\right)\left|a_{i}\right|^{2}
$$

\section{Wireless Standard Channel Models}

Several wireless channel models are used to simulate the radio wave propagation. These channels are currently utilized in industry where each model is convenient for a specific type of environment. Uses of standardized models allow researchers to provide 'apple to apple' comparisons of the obtained results. The considered channels PDPs that are used for the performance evaluation tasks in this paper are:

\section{A. ITU Channel Models}

The ITU channel models consist of two types of the channels. Table. 1 and Table. 2 show the PDPs and the characteristic of these channels. Theses Tables indicate the relative delay, the average power for the taps of the multi-path channel and the RMS delay spread based on the ITU recommendation.

Table 1. Power Delay Profile of ITU-A Channel

\begin{tabular}{|c|c|c|c|c|c|c|}
\hline \multirow{2}{*}{$\begin{array}{l}\text { Tap } \\
\text { No. }\end{array}$} & \multicolumn{2}{|c|}{ Indoor A } & \multicolumn{2}{|c|}{ Pedestrian A } & \multicolumn{2}{|c|}{ Vehicular A } \\
\hline & $\begin{array}{c}\text { Delay } \\
\text { (ns) }\end{array}$ & $\begin{array}{c}\text { Power } \\
(\mathrm{dB})\end{array}$ & $\begin{array}{c}\text { Delay } \\
(\mathrm{ns})\end{array}$ & $\begin{array}{c}\text { Power } \\
(\mathrm{dB})\end{array}$ & $\begin{array}{c}\text { Delay } \\
(\mathrm{ns})\end{array}$ & $\begin{array}{c}\text { Power } \\
(\mathrm{dB})\end{array}$ \\
\hline 1 & 0 & 0 & 0 & 0 & 0 & 0 \\
\hline 2 & 50 & -3.0 & 110 & -9.7 & 310 & -1.0 \\
\hline 3 & 110 & -10.0 & 190 & -19.2 & 710 & -9.0 \\
\hline 4 & 170 & -10.0 & 410 & -22.8 & 1090 & -10.0 \\
\hline 5 & 290 & -26.0 & NA & NA & 1730 & -15.0 \\
\hline 6 & 310 & -32.0 & NA & NA & 2510 & -20.0 \\
\hline & \multicolumn{2}{|c|}{$\tau_{R M S}=35 n s$} & \multicolumn{2}{|c|}{$\tau_{R M S}=45 n s$} & \multicolumn{2}{|c|}{$\tau_{R M S}=370 \mathrm{~ns}$} \\
\hline
\end{tabular}

Table 2. Power Delay Profile of ITU-B Channel

\begin{tabular}{|c|c|c|c|c|c|c|}
\hline \multirow{2}{*}{$\begin{array}{l}\text { Tap } \\
\text { No. }\end{array}$} & \multicolumn{2}{|c|}{ Indoor B } & \multicolumn{2}{|c|}{ Pedestrian B } & \multicolumn{2}{|c|}{ Vehicular B } \\
\hline & $\begin{array}{c}\text { Delay } \\
\text { (ns) }\end{array}$ & $\begin{array}{c}\text { Power } \\
(\mathrm{dB})\end{array}$ & $\begin{array}{c}\text { Delay } \\
(\mathrm{ns})\end{array}$ & $\begin{array}{c}\text { Power } \\
(\mathrm{dB})\end{array}$ & $\begin{array}{c}\text { Delay } \\
(\mathrm{ns})\end{array}$ & $\begin{array}{c}\text { Power } \\
(\mathrm{dB})\end{array}$ \\
\hline 1 & 0 & 0 & 0 & 0 & 0 & -2.5 \\
\hline 2 & 100 & -3.6 & 200 & -0.9 & 300 & 0 \\
\hline 3 & 200 & -7.2 & 800 & -4.9 & 8900 & -12.8 \\
\hline 4 & 300 & -10.8 & 1200 & -8.0 & 12900 & -10.0 \\
\hline 5 & 500 & -18.0 & 2300 & -7.8 & 17100 & -25.2 \\
\hline \multirow[t]{2}{*}{6} & 700 & -25.2 & 3700 & -23.9 & 20000 & -16.0 \\
\hline & \multicolumn{2}{|c|}{$\tau_{R M S}=100 n s$} & \multicolumn{2}{|c|}{$\tau_{R M S}=750 n s$} & \multicolumn{2}{|c|}{$\tau_{R M S}=4000 \mathrm{~ns}$} \\
\hline
\end{tabular}

\section{B. EITU Channel Models}

The Extended ITU channel model for LTE was called Extended Pedestrian-A (EPA), Extended Vehicular-A (EVA), and Extended Typical Urban (ETU). Table.3 shows the PDPs and the characteristic of this channel. This Table indicates the channel models parameters such as the excess delay, the average power and the RMS delay spread.

\section{3GPP Channel Models}

The 3 GPP channel model consists of urban micro-cell and urban macro-cell channel model. Table.4 shows the tap delay, the average power and the RMS delay spread for these channel models.

Table 3. Power Delay Profile of EITU Channel

\begin{tabular}{|c|c|c|c|c|c|c|}
\hline \multirow{2}{*}{$\begin{array}{l}\text { Tap } \\
\text { No. }\end{array}$} & \multicolumn{2}{|c|}{ EPA } & \multicolumn{2}{|c|}{ EVA } & \multicolumn{2}{|c|}{ ETU } \\
\hline & $\begin{array}{c}\text { Delay } \\
\text { (ns) }\end{array}$ & $\begin{array}{c}\text { Power } \\
(\mathrm{dB})\end{array}$ & $\begin{array}{c}\text { Delay } \\
(\mathrm{ns})\end{array}$ & $\begin{array}{c}\text { Power } \\
\text { (dB) }\end{array}$ & $\begin{array}{c}\text { Delay } \\
\text { (ns) }\end{array}$ & $\begin{array}{c}\text { Power } \\
\text { (dB) }\end{array}$ \\
\hline 1 & 0 & 0 & 0 & 0 & 0 & -1.0 \\
\hline 2 & 30 & -1.0 & 30 & -1.5 & 50 & -1.0 \\
\hline 3 & 70 & -2.0 & 150 & -1.4 & 120 & -1.0 \\
\hline 4 & 90 & -3.0 & 310 & -3.6 & 200 & 0.0 \\
\hline 5 & 110 & -8.0 & 370 & -0.6 & 230 & 0.0 \\
\hline 6 & 190 & -17.2 & 710 & -9.1 & 500 & 0.0 \\
\hline 7 & 410 & -20.8 & 1090 & -7.0 & 1600 & -3.0 \\
\hline 8 & NA & NA & 1730 & -12 & 2300 & -5.0 \\
\hline \multirow[t]{2}{*}{9} & NA & NA & 2510 & -16.9 & 5000 & -7.0 \\
\hline & \multicolumn{2}{|c|}{$\tau_{R M S}=45 n s$} & \multicolumn{2}{|c|}{$\tau_{R M S}=357 n s$} & \multicolumn{2}{|c|}{$\tau_{R M S}=991 \mathrm{~ns}$} \\
\hline
\end{tabular}

Table 4. Power Delay Profile of 3GPP Channel

\begin{tabular}{|c|c|c|c|c|}
\hline \multirow{2}{*}{$\begin{array}{c}\text { Tap } \\
\text { No. }\end{array}$} & \multicolumn{2}{|c|}{ Urban Macro } & \multicolumn{2}{c|}{ Urban Micro } \\
\cline { 2 - 5 } & Delay (ns) & $\begin{array}{c}\text { Power } \\
(\mathrm{dB})\end{array}$ & $\begin{array}{c}\text { Delay } \\
(\mathrm{ns})\end{array}$ & $\begin{array}{c}\text { Power } \\
(\mathrm{dB})\end{array}$ \\
\hline 1 & 0 & 0 & 0 & 0 \\
\hline 2 & 0.36 & -2.22 & 0.28 & -1.27 \\
\hline 3 & 0.25 & -1.72 & 0.20 & -2.72 \\
\hline 4 & 1.04 & -5.72 & 0.66 & -4.30 \\
\hline 5 & 2.7 & -9.05 & 0.81 & -6.01 \\
\hline 6 & 4.59 & -12.50 & 0.92 & -8.43 \\
\hline & \multicolumn{2}{|c|}{$\tau_{R M S}=850 n s$} & \multicolumn{2}{c}{$\tau_{R M S}=295 \mathrm{~ns}$} \\
\hline
\end{tabular}

\section{Simulation Results and Discussion}

The focus of this study is to give insight on the choice of an optimal CP length over different wireless channel models.

\section{A. Performance Evaluation of SINR for Varying CP Length}

SINR expressed in Eq. (2) is used to evaluate some specific PDPs such as ITU-A, ITU-B, EITU, 3GPP and the exponential decaying PDPs. Fig. 3, 4, 5 and 6 compare the effect of the CP length on the received SINR over ITU-A, ITU-B, EITU and 3GPP against exponential decaying PDPs with corresponding RMS delay spread. These figures indicate that SINR in $\mathrm{dB}$ is plotted as function of the CP length. It can be observed that SINR value increases as the CP length is increased. It can be seen clearly that SINR has different curves even though the RMS delay spreads are of equal value. For all the curves in these figures, the maximum SINR corresponding to the received SNR is occurred when the $\mathrm{CP}$ length exceeds the maximum excess delay spread of 
the channel due to eliminating the multipath fading. Therefore; increasing the SNR will raise the SINR levels resulting in a larger $\mathrm{CP}$ length requirement. We can demonstrate from these figures that the maximum SINR occurs at the different optimal CP length due to the variable RMS delay spread. Therefore, the larger RMS delay spread required a larger $\mathrm{CP}$ length to get the maximum SINR and vice versa.

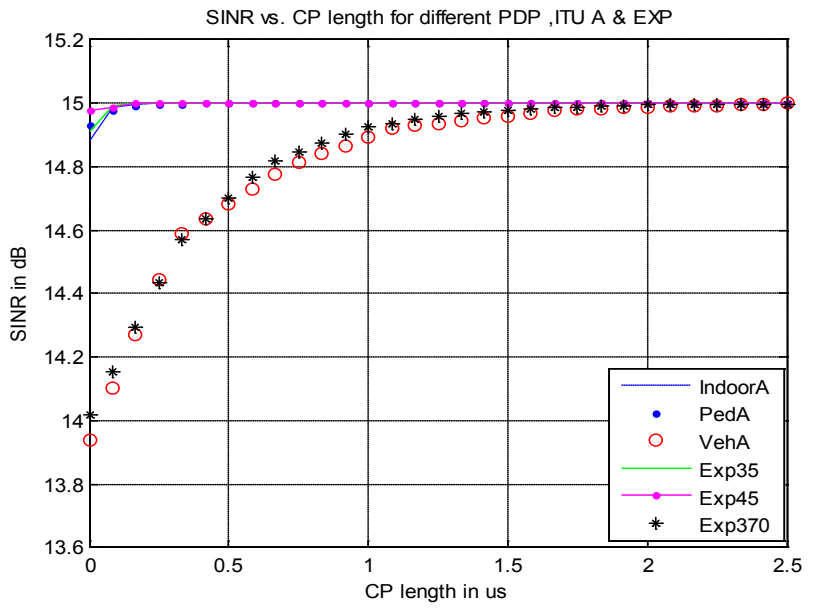

Figure 3. SINR vs. CP length for different PDP $\{$ ITU-A \& EXP

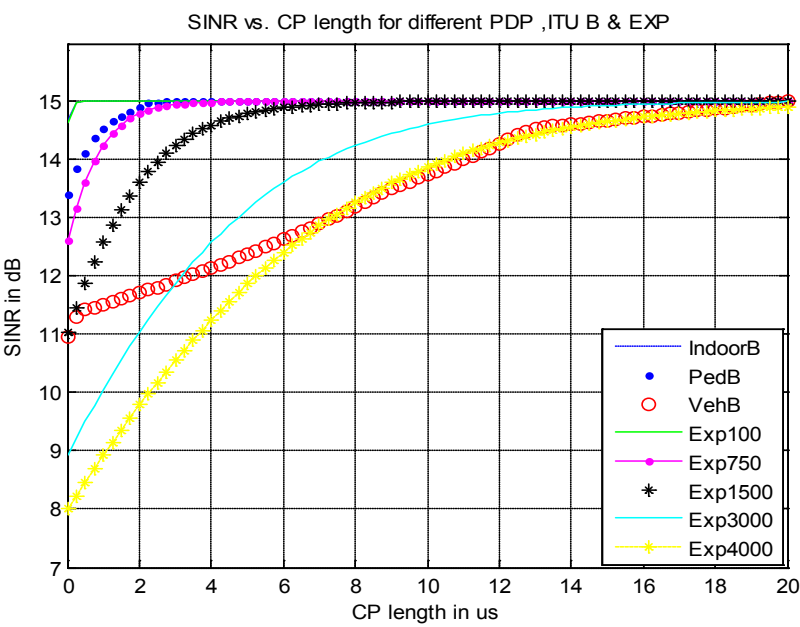

Figure 4. SINR vs. CP length for different PDP $\{$ ITU-B \& EXP $\}$

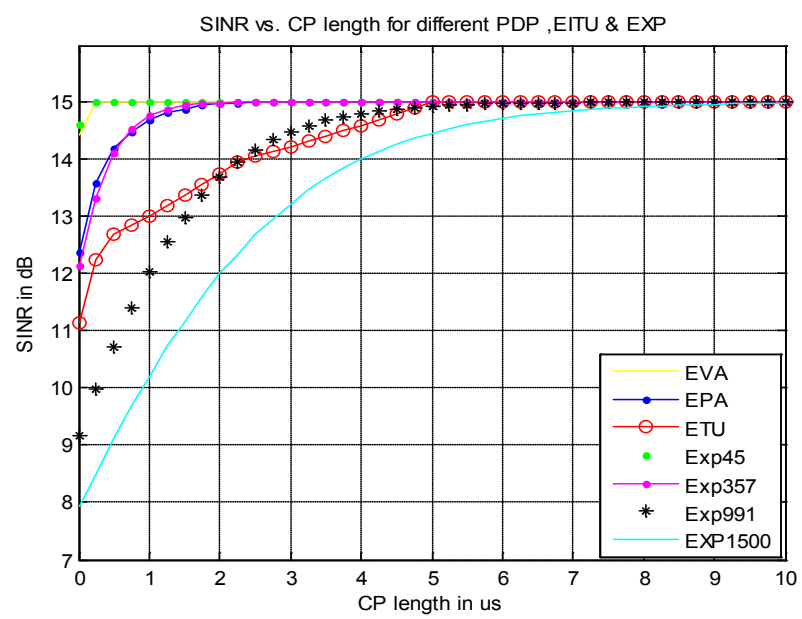

Figure 5. SINR vs. CP length for different PDP $\{$ EITU \& EXP $\}$

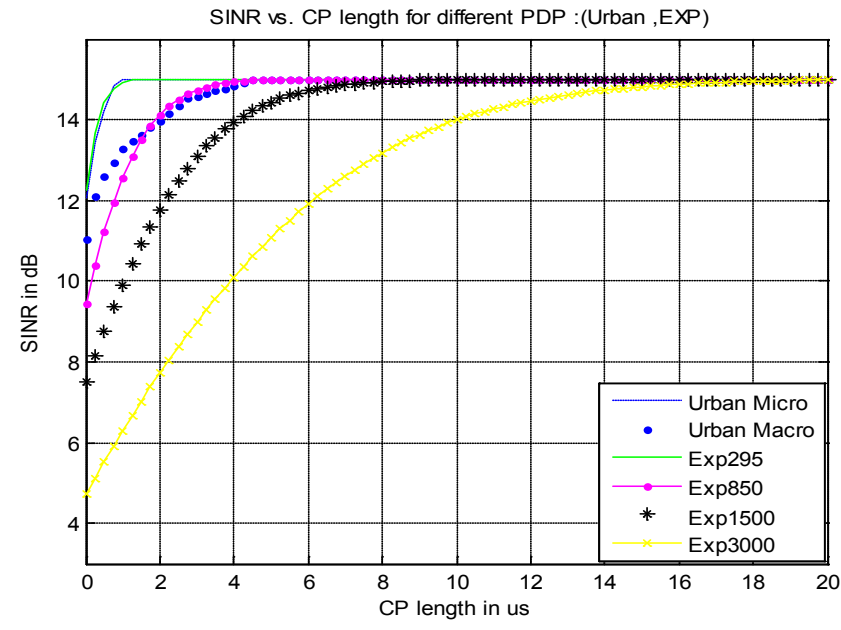

Figure 6. SINR vs. CP length for different PDP $\{3$ GPP \& EXP $\}$

\section{B. Effect of CP Length on OFDM System Spectral Efficiency}

A conducted study giving insight on the impact of the $\mathrm{CP}$ length on the capacity of a base-band synchronized OFDM system. The capacity is used to evaluate the system and it is measured in bits per second per hertz. This study indicates how the CP length affects Spectral Efficiency Loss (SEL) and Signal to Interference and Noise Ratio (SINR) where SINR is mapped to capacity through Shannon's formula [14]. The optimum CP length maximizes the capacity for a given set of system parameters. The system parameters that will be considered in this section are Signal to Noise ratio (SNR) at the receiver side, OFDM useful symbol length and different channels with different PDPs.

As seen previously, maximizing SINR causes high BER at the receiver side, which requires a larger $\mathrm{CP}$ length, but utilizing a larger $\mathrm{CP}$ length extends the transmission time required. Therefore, an optimization of the SINR is required and can be obtained from Shannon's capacity theorem. We used an approach similar to [14] where SINR is mapped to capacity in b/s/Hz through Shannon's Capacity theorem. In doing this we accounted for a link adaptation loss $\propto_{\text {loss }}$ and a maximum bandwidth efficiency corresponding to 64QAM modulation. It follows that the capacity model over useful OFDM symbol length for the $p^{\text {th }}$ channel profile is given by:

$$
\mathrm{C}\left(\mathrm{T}_{\mathrm{g}}\right)=(1-\mathrm{SEL}) * \min \left\{\log _{2}\left(1+\alpha_{\text {loss }} \operatorname{SINR}\left(\mathrm{T}_{\mathrm{g}}\right)\right), 6\right\}
$$

Where SEL is the Spectral Efficiency Loss (CP length / Total OFDM symbol length) and $\propto_{\text {loss }}$ is the degradation factor which has a value of 0.4 for this study as it is used in [14].

\section{1- Effect of SNR on the CP Length}

Equation (2) shows that the SINR depends on the received SNR. The received SNR becomes equal to the SINR as the CP length exceeds the maximum excess delay spread of the channel impulse response as shown previously. Figs. 7 shows the influence of the received SNR on the CP length over Ped-B PDP. It can be observed that the effect of increasing the $\mathrm{CP}$ length at receiver is more pronounced at 
high SNR, whereas at low SNR its effect is insignificant. I.e. at $31 \mathrm{~dB}$ there is a difference of $1.5 \mathrm{bit} / \mathrm{s} / \mathrm{Hz}$ in capacity for $\mathrm{CP}$ length varying between 0 and $1.6 \mu \mathrm{s}$, but at $1 \mathrm{~dB}$ the variation is about $0.1 \mathrm{bit} / \mathrm{s} / \mathrm{Hz}$ in capacity for the same interval. Therefore; we can conclude that increasing the received SNR increases the required $\mathrm{CP}$ length, while a decrease in received SNR tolerates a lower CP requirement.

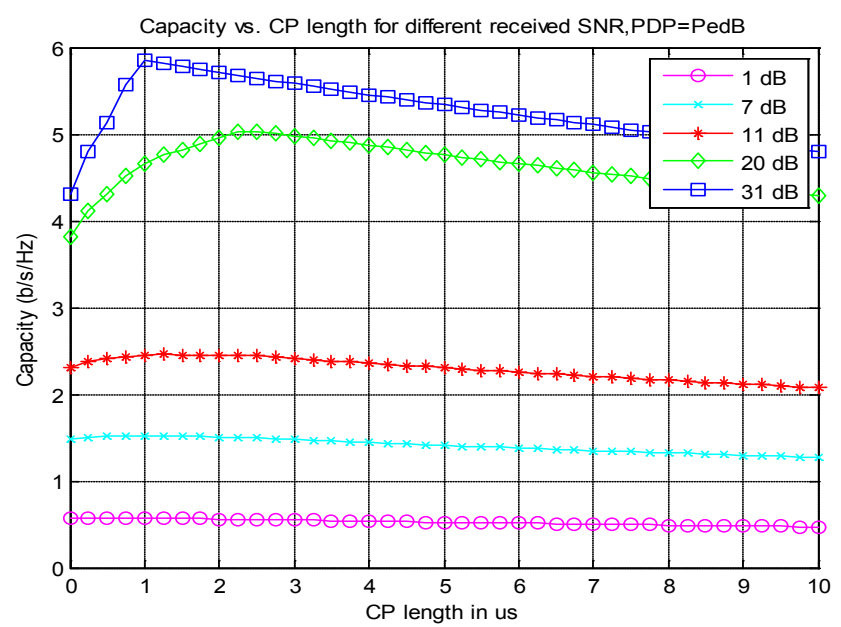

Figure 7. Capacity vs. CP length for different received SNR, PDP=Ped B

\section{2- Effect of the Symbol Duration on the CP Length}

The effect of the useful symbol length $\left(T_{u}\right)$ on the $\mathrm{CP}$ length over EPA PDP is studied in Fig. 8. It can be seen that the capacity of longer useful symbol length is minimally affected by $\mathrm{CP}$ length changes, but the capacity of shorter useful symbol length suffers more variation with changes in $\mathrm{CP}$ length. I.e. if $\mathrm{CP}=1.4 \mu \mathrm{s}$ then with $T_{u}=160 \mu \mathrm{s}$ the capacity is decreased by $0.1 \mathrm{bit} / \mathrm{s} / \mathrm{Hz}$ from the maximum achievable performance, while with $T_{u}=10 \mu$ s the loss would be more than $0.7 \mathrm{bit} / \mathrm{s} / \mathrm{Hz}$. Therefore; the longer symbol length is preferable to shorter symbol length.

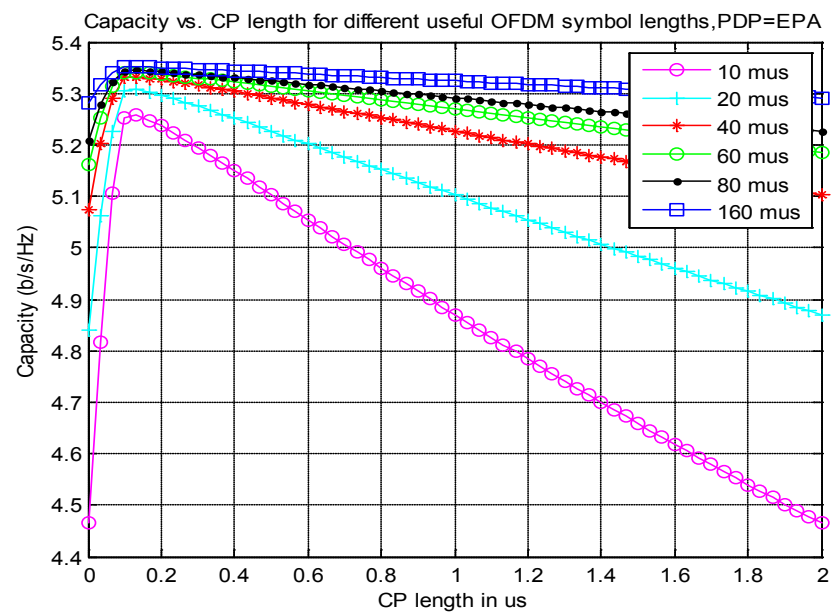

Figure 8. Capacity vs. CP length for different $\boldsymbol{T}_{\boldsymbol{u}}, \mathrm{PDP}=\mathrm{EPA}$.

\section{3- Effect of the CP Length on the Different Power Delay Profiles:}

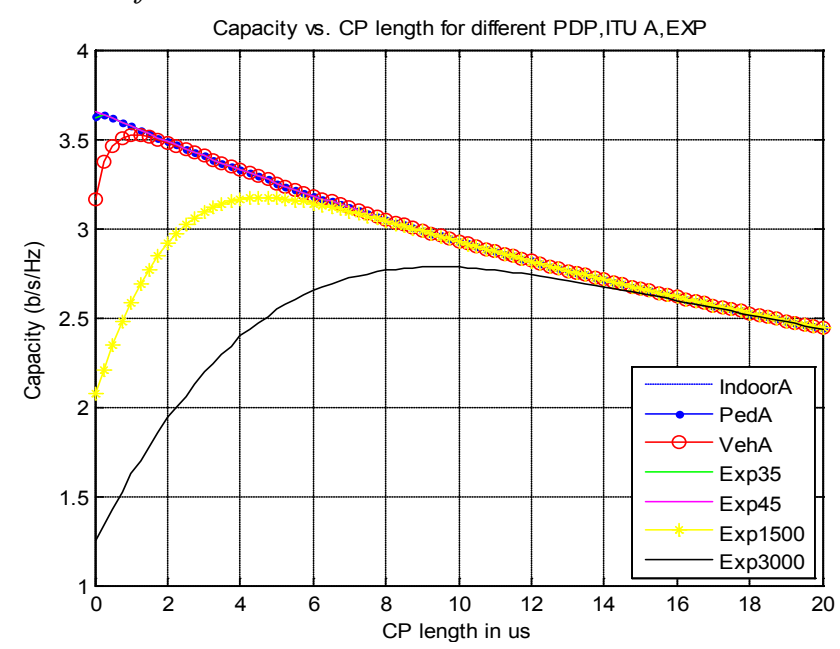

Figure 9. Influence of the ITU-A PDP \& EXP PDP on CP length.

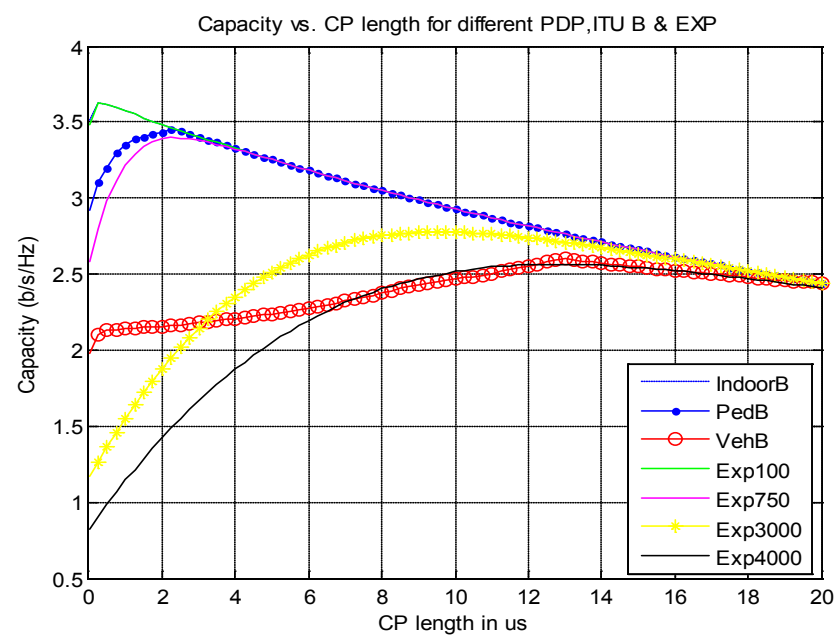

Figure 10. Influence of the ITU-B PDP \& EXP PDP on CP length.

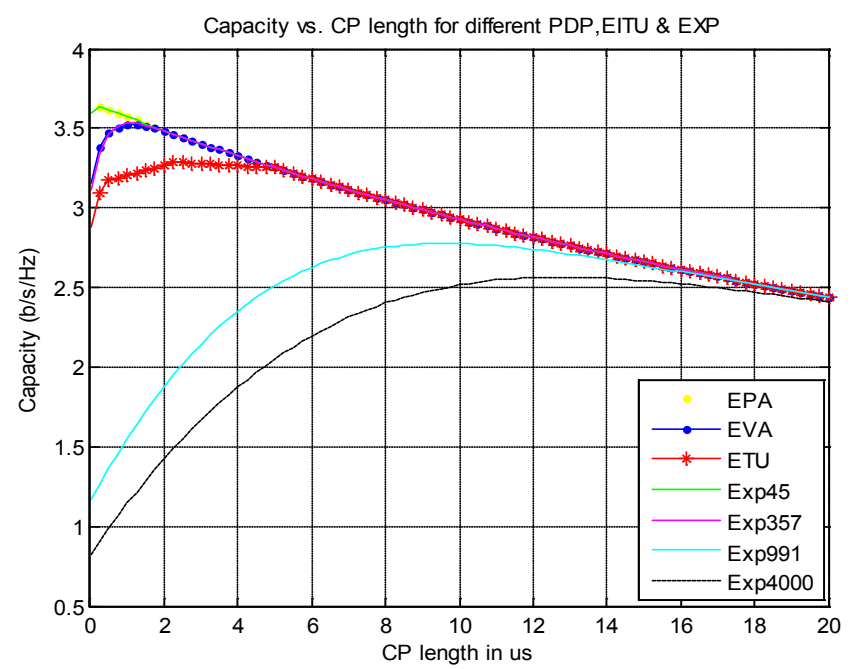

Figure 11. Influence of the EITU PDP \& EXP PDP on CP length 


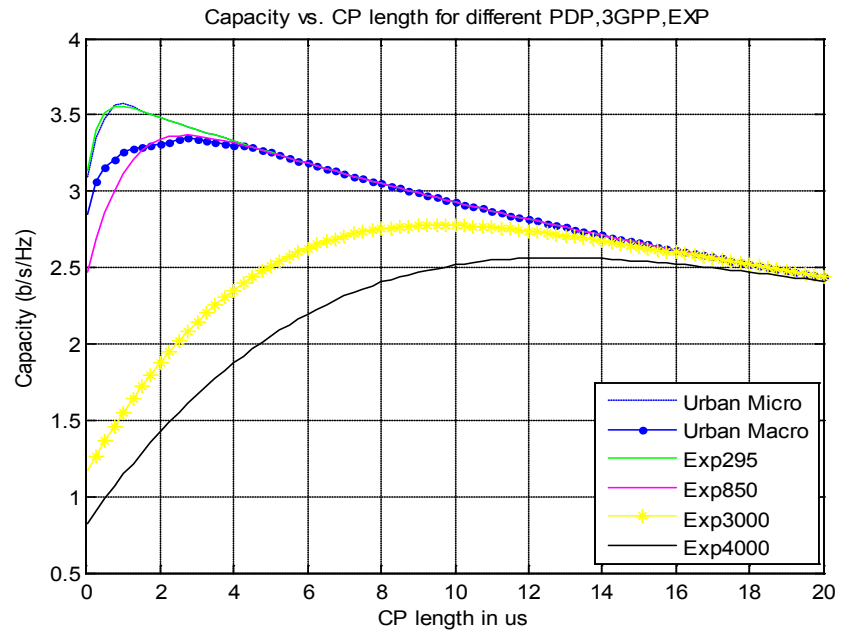

Figure 12. Influence of the ITU-A PDP \& EXP PDP on CP length.

Figures 9, 10, 11 and 12 show the effect of varying the $\mathrm{CP}$ length on different channel PDPs. In these figures, the CP length varies from one PDP to another based on the RMS delay spread. Exponentially decaying PDPs are plotted with different RMS delay spread. It is noted that the optimal CP length for exponential decaying PDPs increases as the RMS delay spread is increased. When comparing the exponential decaying PDPs with standard PDPs with equal RMS delay spread, we will see that the optimal CP length can significantly vary from profile to profile. As can be seen in Fig.9 and Fig.10, the indoor channel has the smallest CP length while the vehicular channel has the largest $\mathrm{CP}$ length because the vehicular channel has a large delay spread. In Fig.11, the ETU PDP has larger CP length as compared to EPA PDP and EVA PDP, because the ETU PDP has a larger delay spread. In Fig.12, the macro-cell PDP required a larger $\mathrm{CP}$ length than micro-cell PDP, because the macro-cell has a larger delay spread. These figures indicate that the CP length increases as the RMS delay spread is increased. One of the methods used to estimate the optimal $\mathrm{CP}$ length is based on the RMS delay spread; according to this approach the $\mathrm{CP}$ length is estimated and it is given as: $\left(C P=\alpha^{*} R M S\right)$ where $\alpha$ is the constant in the range between ( 2.5 to 3.5 ). This conforms the findings of a previous study by Arslan who found that the $(C P=\beta * R M S))$ where $\beta$ is the constant in the range between (2 to 4$)$ [15]. It can also be observed from these figures the estimated $\mathrm{CP}$ maximizes the system capacity, but increasing the $\mathrm{CP}$ length beyond this optimum CP length results in decreasing the system capacity.

\section{Mathematical Relationship between the $\mathrm{CP} \&$ the RMS Delay Spread of the Channel}

As we mentioned earlier the $\mathrm{CP}$ can be estimated based on the RMS delay spread of the channel. In this study the SINR is applied to the PDP and the CP is calculated based on the RMS delay spread. It is found that $C P=\alpha^{*} R M S$ where $\alpha$ is the constant in the range between (2.5 to 3.5$)$ optimizes the system's performance. Fig 13,14 and 15 show the mathematical relationship between the CP and the RMS delay spread of different multipath channel. It can be seen clearly from these figures that the $\mathrm{CP}$ length increases as the RMS delay spread of the channel is increased which indicates a linear relationship exists between them. Obviously the RMS delay spread is not fixed in the mobile wireless communication and its value changes depending on the terrain, clutter, antenna directivity and other factors related to propagation environment. The conventional OFDM system uses a fixed and large CP length which increases the overhead, but if one estimates the CP length based on the RMS delay spread the overhead will be reduced. Therefore; the CP length needs to be adjusted to the environment so that the mobile battery power and scarce spectrum can be conserved when the delay spread is small. Furthermore the system performance will be maintained by reducing the ISI and ICI when the delay spread is large. From these figures, it is evident that channels such as the Indoor A, $\mathrm{B}$ and EPA with small RMS delay spread require small CP length, whereas the channel such as the vehicular B and ETU channel with large RMS delay spread requires large CP length. It can also be seen the channel with exponentially decaying PDPs required a large CP length as RMS delay spread is increased.

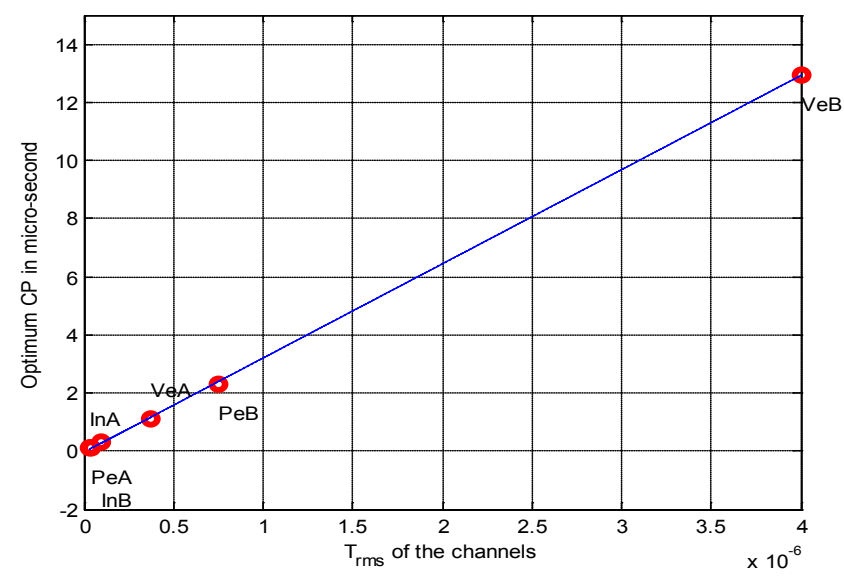

Figure 13. CP lengths vs. RMS delay spread for ITU-A, \& ITU-B

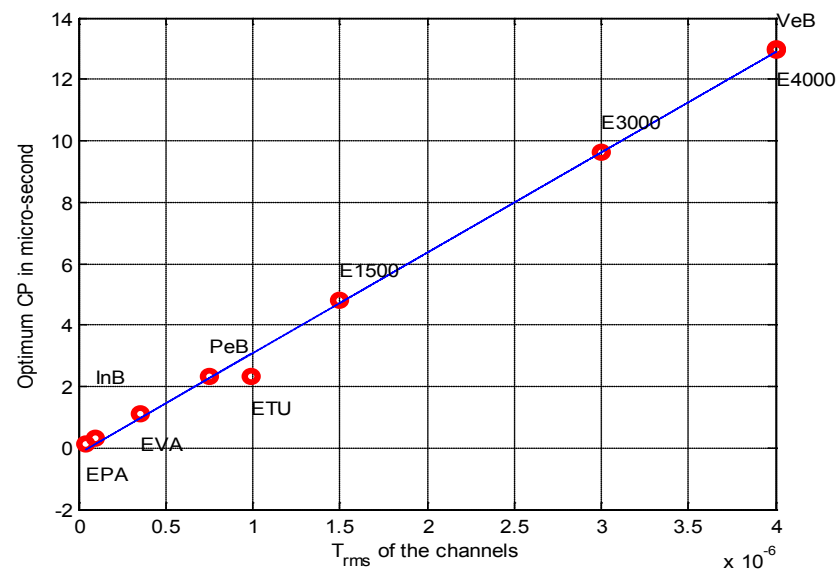

Figure 14. CP lengths vs. RMS delay spread for ITU-B, EITU \& EXP 


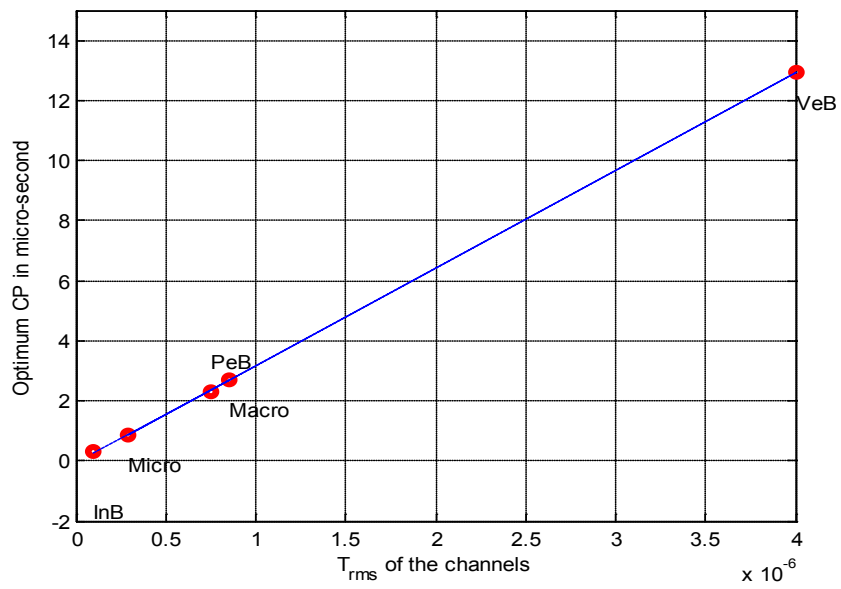

Figure 15. CP lengths vs. RMS delay spread for ITU-B, \& 3GPP

As aforementioned, the proposed $\mathrm{CP}$ model is estimated based on RMS delay spread, and it is found that $C P=\alpha * R M S$ represent a mathematical formula to calculate the estimated $\mathrm{CP}$ length over different wireless channel models. According to this approach, the Table. 5 shows the optimal parameters associated with this analysis to determine the optimal CP length over different multipath channels.

Table 5. The values of the CP length for different channel models according to the RMS delay spread method.

\begin{tabular}{|c|c|c|c|}
\hline Channel Type & RMS delay & $\begin{array}{c}\text { Beta } \\
\text { value }\end{array}$ & $\mathbf{C P}=\boldsymbol{\beta} * \boldsymbol{\tau}_{\mathrm{RMS}}$ \\
\hline EXP & variable & 3 & variable \\
\hline Indoor A & $\tau_{R M S}=35 \mathrm{~ns}$ & 3.17 & $0.111 \mu \mathrm{s}$ \\
\hline Pedestrian A & $\tau_{R M S}=45 \mathrm{~ns}$ & 2.7 & $0.121 \mu \mathrm{s}$ \\
\hline Vehicular A & $\tau_{R M S}=370 \mathrm{~ns}$ & 3.04 & $1.127 \mu \mathrm{s}$ \\
\hline & & & \\
\hline Indoor B & $\tau_{R M S}=100 \mathrm{~ns}$ & 3 & $0.3 \mu \mathrm{s}$ \\
\hline Pedestrian B & $\tau_{R M S}=750 \mathrm{~ns}$ & 3.1 & $2.3 \mu \mathrm{s}$ \\
\hline Vehicular B & $\tau_{R M S}=4000 \mathrm{~ns}$ & 3.4 & $13.6 \mu \mathrm{s}$ \\
\hline & & & \\
\hline EPA & $\tau_{R M S}=45 \mathrm{~ns}$ & 2.7 & $0.121 \mu \mathrm{s}$ \\
\hline EVA & $\tau_{R M S}=357 \mathrm{~ns}$ & 3.15 & $1.124 \mu \mathrm{s}$ \\
\hline ETU & $\tau_{R M S}=991 \mathrm{~ns}$ & 2.52 & $2.5 \mu \mathrm{s}$ \\
\hline & & & \\
\hline Urban Macro & $\tau_{R M S}=850 \mathrm{~ns}$ & 3.05 & $2.4 \mu \mathrm{s}$ \\
\hline Urban Micro & $\tau_{R M S}=295 \mathrm{~ns}$ & 2.88 & $0.8 \mu \mathrm{s}$ \\
\hline
\end{tabular}

\section{Conclusions}

An adaptive OFDM system with variable CP length over different channel models with different PDPs was described in this paper. We presented a method to estimate the CP length based on the RMS delay spread of the channel. According to this approach, the CP length is chosen to improve the system performance and to optimize the system capacity by mitigating both ISI and ICI. We demonstrated that the optimal CP length correlates very well with the RMS delay spread of the channel. Therefore, the choice of variable $\mathrm{CP}$ length is preferable to large and fixed CP length because it offers improved spectral efficiency. Furthermore, these simulation results showed that increasing the $\mathrm{CP}$ length beyond the optimum length does not result in further improvement of the system capacity and the system performance.

Finally, it may be concluded that the CP length is an essential aspect of the OFDM system. When the CP selected optimally based on the channel parameters, the overall system performance is improved. This is accomplished through effectively mitigating the dominant problems (ISI and ICI) in the OFDM system.

\section{REFERENCES}

[1] Kaur, Sandeep, and Gurpreet Bharti. "Orthogonal Frequency Division Multiplexing in Wireless Communication Systems: A Review." International Journal of Advanced Research in Computer Engineering \& Technology (IJARCET) 1, no. 3 (2012): pp-125.

[2] Marchetti, Nicola, Muhammad Imadur Rahman, Sanjay Kumar, and Ramjee Prasad. "OFDM: Principles and Challenges." In New Directions in Wireless Communications Research, pp. 29-62. Springer US, 2009.

[3] Prasad, Ramjee. OFDM for wireless communications systems. Artech House, 2004.

[4] J. Li and M. Kavehrad, "Effects of time selective multipath fading on OFDM systems for broadband mobile applications," IEEE Commun. Lett., vol. 3, no. 12, pp. 332-334, 1999.

[5] Y. Zhang and H. Liu, "Impact of time selective fading on the performance of quasi-orthogonal space-time coded OFDM systems," IEEE Trans. Commun., vol. 54, no. 2, pp. 251-260, 2006.

[6] Shah, D. C., B. U. Rindhe, and S. K. Narayankhedkar. "Effects of cyclic prefix on OFDM system." In Proceedings of the International Conference and Workshop on Emerging Trends in Technology, pp. 420-424. ACM, 2010.

[7] Ness, Reto, Jean-Paul Linnartz, Liesbet Van der Perre, and Marc Engels. "The OFDM Principle." In Wireless OFDM Systems, pp. 33-51. Springer US, 2002.

[8] Payaswini P, and Manjaiah D.H "Analysis of Effect of Cyclic Prefix on Data Rates in OFDM Modulation Techniques" International Journal of Advanced Research Computer and Mathemmatical Sciences (IJARCMS), Vol. 3 ,2012, pp-465-470.

[9] Das, Suvra Sekhar, Frank HP Fitzek, E. D. Carvalho, and Ramjee Prasad. "Variable guard interval orthogonal frequency division multiplexing in presence of carrier frequency offset." In Global Telecommunications Conference, 2005. GLOBECOM'05. IEEE, vol. 5, pp. 5-pp. IEEE, 2005.

[10] Ghosh, Sayani. "Performance Study of WiMAX for Different 
QoS Parameters with Varying Cyclic Prefix." PhD diss., JADAVPUR UNIVERSITY KOLKATA, 2011.

[11] Zhang, Zhao-yang, and Li-feng Lai. "A novel OFDM transmission scheme with length-adaptive Cyclic Prefix." Journal of Zhejiang University SCIENCE 5, no. 11 (2004): 1336-1342.

[12] Lim, Chiwoo, Youngbin Chang, Jaeweon Cho, Panyuh Joo, and Hyeonwoo Lee. "Novel OFDM transmission scheme to overcome caused by multipath delay longer than cyclic prefix." In Vehicular Technology Conference, 2005. VTC 2005-Spring. 2005 IEEE 61st, vol. 3, pp. 1763-1767. IEEE,
2005.

[13] Nolan, Keith E. "Reconfigurable OFDM systems." PhD diss., Trinity College Dublin., 2005.

[14] Batariere, Mickael, Kevin Baum, and Thomas P. Krauss. "Cyclic prefix length analysis for 4G OFDM systems." In Vehicular Technology Conference, 2004. VTC2004-Fall. 2004 IEEE 60th, vol. 1, pp. 543-547. IEEE, 2004.

[15] Arslan, Hüseyin, and Tevfik Yucek. "Delay spread estimation for wireless communication systems." In Computers and Communication, 2003.(ISCC 2003). Proceedings. Eighth IEEE International Symposium on, pp. 282-287. IEEE, 2003 